Isabelle Dusanter-Fourt Patrick Mayeux Sylvie Gisselbrecht

\section{ADRESSE}

I. Dusanter : chargée de recherche à l'INSA.P. Mayeux : chargé de recherche au Cnrs. S. Gisselbrecht : directeur de recherche à l'Inserm, Inserm U.363, ICGM, hôpital Cochin, 27, rue du Faubourg-Saint-Jacques, 75014 Paris, France.

\title{
Transduction du signal par les récepteurs de cytokines
}

Les cytokines règlent la prolifération et la différenciation des cellules hématopoiétiques et notamment la réponse immunitaire. Leurs récepteurs sont composés de chaînes communes et de chaînes spécifiques portant le site de liaison de la cytokine. La fixation d'une cytokine sur son récepteur entraîne la formation d'un complexe multimérique qui, à son tour, recrute des tyrosine kinases de divers types : des kinases de la famille Jak, qui phosphorylent les facteurs de transcription de type Stat dont les cibles nucléaires sont encore mal connues ; d'autres kinases, dont certaines appartiennent à la famille Src, qui transmettent le signal mitogène en empruntant d'autres voies, en particulier la voie de Ras et des MAP kinases. Le récepteur est généralement lui-même phosphorylé, créant ainsi des points de fixation pour des protéines de transmission à domaine $\mathrm{SH}$, dont une tyrosine phosphatase qui pourrait être impliquée dans la mise au repos du récepteur.

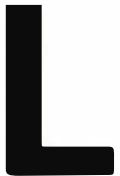

e clonage des $\mathrm{ADN}$ complémentaires (ADNc) des récepteurs de nombreuses interleukines et facteurs de croissance hématopoïétiques a révélé que la plupart de ces récepteurs présentent des analogies structurales importantes permettant de les réunir dans une même famille sous le terme de "récepteurs de cytokines".

\section{Les récepteurs} de cytokines : une famille qui grandit

Cette famille regroupe des récepteurs de facteurs de croissance hématopoïétiques tels que les récepteurs de l'interleukine 2 (IL2), IL3,
IL4, IL5, IL6, IL7, IL9, les récepteurs du GM-CSF, du G-CSF, de l'érythropoïétine (Epo), le produit du gène c-mpl dont le ligand semble impliqué dans la régulation de la mégacaryocytopoïèse $\left(\mathrm{m} / \mathrm{s} n^{\circ} 1\right.$, vol. 10, p. 114, $n^{\circ} 8 / 9$, vol. 10 , p. 884), les récepteurs du LIF [1], de l'oncostatine M (OSM), mais aussi des récepteurs hormonaux tels que les récepteurs de l'hormone de croissance $(\mathrm{GH})$, de la prolactine (PRL) et le récepteur du facteur neurotrophique ciliaire, le CNTF [2]. De plus, bien que les gènes des chaînes polypeptidiques fixant l'ILl1 et l'IL13 ne soient pas encore caractérisés, on sait que ces récepteurs comportent au moins une chaîne appartenant à la famille des récepteurs de cytokines (gp130 pour le 


\section{RÉFÉRENCES}

1. Jacques Y, Anegon I, Hallet MM, Godard A, Moisan JP, Soulillou JP. Les multiples facettes de la cytokine HILDA/LIF. médecine/sciences 1992 ; 8 : 93949.

2. Bazan JF. Structural design and molecular evolution of a cytokine receptor superfamily. Proc Natl Acad Sci USA 1990 superfamily.
$87: 6934-8$.

3. Yin T, Taga T, Tsang ML, Yasukawa $K$ Kishimoto T, Yang YC. Involvement of IL-6 signal transducer gp130 in IL-11-mediated signal transduction. J Immunol 1993; 151 : 2555-61.

4. Zurawski SM, Vega FJ, Huyghe B Zurawski G. Receptors for IL-13 and IL-4 are complex and share a novel component that functions in signal transduction. EMBOJ $1993 ; 12: 2663-70$.

5. Gearing DP, Cosman D. Homology of the $\mathrm{p} 40$ subunit of natural killer cell stimulatory factor (NKSF) with the extracellular domain of the interleukin- 6 receptor. Cell 1991; 66:9-10.

6. Kaushansky K, Karplus PA. Hematopoietic growth factors : understanding functional diversity in structural terms. Blood $1993 ; 82: 3229-40$.

7. Lutfalla G, Gardiner K, Proudhon D, Vielh E, Uze G. The structure of the human interferon $\alpha / \beta$ receptor gene. $J$ Biol Chem $1992 ; 267$ : 2802-9.

8. Miyajima A, Mui ALF, Ogorochi T, Sakamaki K. Receptors for granulocytemacrophage colony-stimulating factor interleukin-3, and interleukin-5. Blood $1993 ; 82: 1960-75$.

9. Davis S, Aldrich TH, Stahl N, Pan L, Taga T, Kishimoto T, Ip NY, Yancopoulos GD. LIFRb and gp 130 as heterodimerizing signal transducers of the tripartite CNTF receptor. Science $1993 ; 260: 1805-8$.

10. Murakami M, Hibi M, Nakagawa N, Nakagazwa T, Yasukawa K, Amanishi K. Taga T, Kishimoto T. IL-6 induced homodimerization of gp130 and associated activation of a tyrosine kinase. Science 1993 $260: 1808-10$.

11. Voss SD, Hong R, Sondel PM. Severe combined immunodeficiency, interleukin-2 (IL-2), and the IL-2 receptor : experiment of nature continue to point the way. Blood $1994 ; 83: 626-35$. récepteur de l'ILll et chaîne partagée avec le récepteur de l'IL4 pour le récepteur de l'IL13) [3, 4]. Enfin, par analogie avec la structure du récepteur de l'IL6, il est probable que le récepteur de l'IL12 appartienne lui aussi à cette famille de récepteurs [5].

$\mathrm{Au}$ contraire de leurs récepteurs, les cytokines ne montrent pas d'homologies de séquence primaire permettant de les réunir au sein d'une même famille. Cependant, des études récentes de cristallographie ou de résonance magnétique nucléaire montrent que leur structure spatiale est extrêmement conservée. En effet, la structure de chacune des cytokines étudiée jusqu'à présent comporte quatre hélices $\alpha$ séparées par de courtes boucles non structurées. Il est donc remarquable que des séquences primaires totalement différentes puissent engendrer des structures spatiales similaires [6]. A l'exception du récepteur du CNTF ancré dans la membrane par un glycosyl-phosphatidylinositol (GPI), les récepteurs de cytokines présentent un domaine transmembranaire unique et des analogies de structure dans leur domaine extracellulaire. Ces récepteurs ont en commun, dans leur domaine extracellulaire, un module de 210 acides aminés comportant de nombreux acides aminés conservés, et, en particulier, un motif WSXWS (Trp-Ser-acide aminé quelconque-Trp-Ser) proche du domaine transmembranaire et deux paires de cystéines dans la région $\mathrm{N}$-terminale engagées dans des ponts disulfures intrachaînes nécessaires à la fixation du ligand. Certaines chaînes de récepteurs de cytokines comportent une duplication de ce module de 210 acides aminés, des domaines structuraux supplémentaires de type immunoglobuline (Ig) et/ou des répétitions de type fibronectine (figure 1) mais seul le module récepteur de cytokine est impliqué dans la fixation du ligand. Dans leur domaine intracellulaire, ces récepteurs ne possèdent pas de site de fixation d'ATP ni d'homologie de séquence avec les sérine/ thréonine ou tyrosine kinases. Bien que la séquence en acides aminés des récepteurs des interférons ne contienne pas les motifs caractéristiques des récepteurs de cytokines, ces récepteurs présentent également un seul domaine transmembranaire et sont dépourvus d'activité enzymatique intrinsèque. De plus, les prédictions de structure suggèrent une parenté entre récepteurs des interférons et récepteurs de cytokines [2], et une origine génétique commune a été proposée [7]. Cette hypothèse s'est vue confortée récemment par la découverte de voies de signalisation communes à ces deux familles de récepteurs.

\section{Structure multimérique des récepteurs de cytokines : une redondance structurale et fonctionnelle}

Contrairement aux récepteurs à activité tyrosine kinase qui, pour la plupart, sont constitués d'une seule chaîne polypeptidique et forment des homodimères après fixation de leur ligand, les récepteurs de cytokines ont une structure souvent complexe et comportent une, deux, voire trois chaînes polypeptidiques différentes codées par des gènes distincts. L'expression d'une seule chaîne polypeptidique dans des cellules COS-7 transfectées s'est avérée parfois suffisante pour la formation de récepteurs à affinité relativement élevée, proche de celle observée dans les cellules qui expriment physiologiquement ces récepteurs. Ainsi, le récepteur de l'hormone de croissance, les récepteurs de la prolactine et du G-CSF semblent constitués d'une seule chaîne polypeptidique qui forme des homodimères après fixation de leur ligand respectif. Dans de nombreux cas, cependant, la coexpression de deux chaînes différentes, une chaîne dite a fixant spécifiquement le ligand avec une faible affinité et une chaîne $b$, ne fixant généralement pas le ligand quand elle est exprimée seule, permet l'expression membranaire de récepteurs de forte affinité. Il semble, dans le cas de récepteurs constitués de deux chaînes différentes, que la première chaîne (a) assure la spécificité de reconnaissance alors que la seconde chaîne (b) stabilise les complexes réalisés par la première chaîne et l'hormone et 


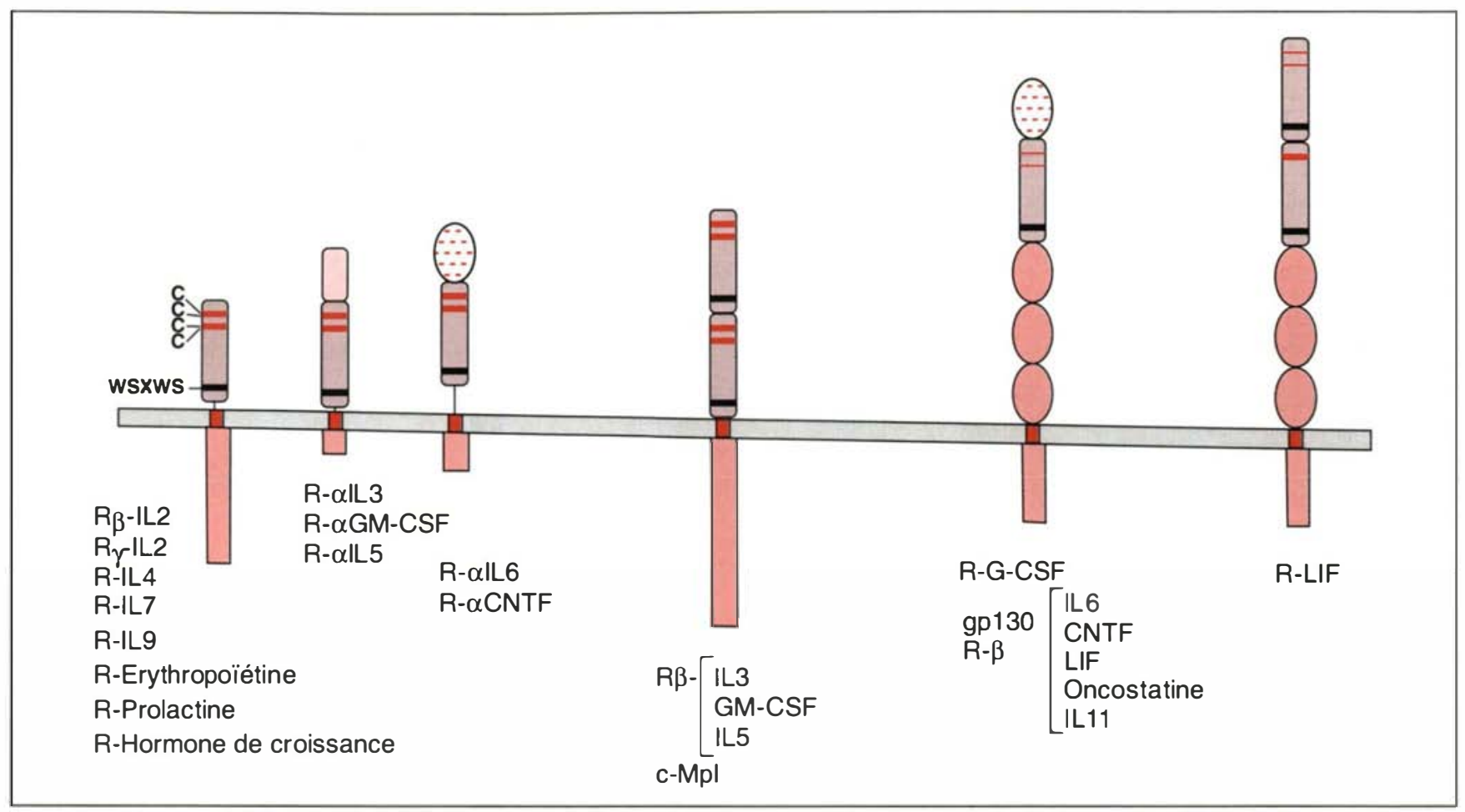

Figure 1. Représentation schématique des différentes chaînes de récepteurs de cytokines. En rose, le domaine "récepteur de cytokines" (210 acides aminés) caractéristique de cette famille et qui comporte 4 cystéines à son extrémité $\mathrm{N}$-terminale et un motif WS XWS à son extrémité C-terminale. Ce motif est dupliqué dans la chaîne $\beta$ commune à I'IL3, le GM-CSF et l'IL5, le produit du gène c-mpl et la chaîne fixant le LIF. En gris, le motif immunoglobuline présent à l'extrémité N-terminale des chaînes $\alpha$ de l'IL6, du CNTF, du récepteur du G-CSF et de la gp130, chaîne partagée par les récepteurs de I'IL6, du CNTF, du LIF, de l'oncostatine et de I'IL11. En bistre, les motifs fibronectines présents dans les récepteurs du LIF, du G-CSF et la gp 130.

participe à la transmission du signal intracellulaire.

De nombreuses cytokines exercent des effets biologiques très proches. Cette redondance peut maintenant s'expliquer par le fait que la même chaîne $\beta$ est souvent partagée par plusieurs récepteurs de cytokines. Ainsi, chez l'homme, les récepteurs de l'IL3, du GM-CSF et de l'IL5 sont composés de deux chaînes, une chaîne $\alpha$ qui est responsable de la spécificité de fixation de chacune de ces trois cytokines et une chaîne $\beta$ commune (KH97). La chaîne $\beta$ participant à la transmission de signaux intracellulaires, il en résulte que l'IL3, l'IL5 et le GM-CSF induisent les mêmes effets biologiques sur des cellules coexprimant les récepteurs de ces trois cytokines, comme les éosinophiles [8]. L'existence d'une chaîne commune, probablement exprimée en quantité limitante, explique aussi $\mathrm{m} / \mathrm{s} n^{\circ} 8 / 9$ vol. 10, aoút-septembre 94 qu'il existe une compétition entre le GM-CSF et l'IL3 pour la formation de récepteurs de forte affinité dans les cellules coexprimant les deux types de récepteurs. Chez la souris, la situation est compliquée du fait d'une duplication récente $\mathrm{du}$ gène codant pour la chaîne $\beta$ : il existe, en effet, dans cette espèce deux chaînes $\beta$, AIC2A et AIC2B, et si chacune d'entre elles peut s'associer à la chaîne $\alpha$ du récepteur de l'IL3, seule AIC2B peut s'associer aux chaînes $\alpha$ des récepteurs du GM-CSF et de l'IL5 (figure 2A) [8].

Un autre groupe de récepteurs, les récepteurs de l'IL6, du CNTF, du LIF, de l'oncostatine et de l'ILll partagent une chaîne $\beta$ commune, la gpl30. Cette chaîne, définie initialement comme la chaîne $\beta$ du récepteur de l'IL6, ne fixe ni l'IL6, ni le CNTF, ni le LIF, mais fixe l'oncostatine (OSM) avec une faible affinité. La gp130 joue un rôle majeur dans la transduction du signal et rend compte de la similitude des effets biologiques de ce groupe de cytokines sur leurs cellules cibles, qu'elles soient hématopoïétiques, hépatiques, musculaires ou d'origine neuronale [1]. Le domaine intracytoplasmique de la chaîne $\alpha$ du récepteur de l'IL6 n'est pas nécessaire à la signalisation par l'IL6 : le domaine extracellulaire de cette chaîne, exprimé sous forme de protéine soluble, s'associe, en présence d'IL6, au domaine extracellulaire de la gp130 et induit, tout au moins in vitro, les effets biologiques de l'IL6 sur des cellules exprimant la seule gp130. Une deuxième chaîne, de 190 $\mathrm{kDa}$ (gpl90), dont la structure est proche de celle de la gpl30 et qui fixe le LIF, est elle aussi commune aux récepteurs du LIF, de l'oncostatine et du CNTF et est nécessaire à la signalisation par ces trois cytokines. 


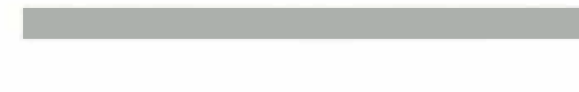

\section{RÉFÉRENCES}

12. Noguchi M, Nakamura Y, Russell S, Ziegler SF, Tsang M, Cao X, Leonard W. Interleukin-2 receptor chain : a functional component of the interleukin-7 receptor. Science 1993 ; 262 : 1877-80.

13. Kondo $M$, Takeshita T, Ishii N, Nakamura M, Watanabe $S$, Arai $K$, Sugamura K. Sharing of the interleukin-2 (IL-2) receptor gamma chain between receptors for IL-2 and IL-4. Science 1993 ; $262: 1874-7$.

14. Russell S, Keegan AD, Harada N Nakamura Y, Noguchi M, Leland P Friedmann M, Miyajima A, Puri RK, Paul WE, Leonard W. Interleukin-2 receptor $\gamma$ chain : a functional component of the interleukin-4 receptor. Science 1993 ; 262 : 1880-2.

15. D'Andrea A, Yoshimura A, Youssufian H, Zon LI, Koo JW, Lodish HF The cytoplasmic region of the erythropoietin receptor contains nonoverlapping positive and negative growth-regulatory domains. Mol Cell Biol $1991 ; 11: 1980-7$.

16. Mayeux P, Lacombe C, Casadevall N, Chrétien S, Dusanter I, Gisselbrecht S Structure of the murine erythropoietin receptor complex : characterization of the erythropoietin cross-linked proteins. J Biol Chem $1991 ; 266: 23380-5$

17. Chiba T, Nagata Y, Kishi A, Sakamaki K, Miyajima A, Yamamoto M, Engel JD, Todokoro K. Induction of erythroidspecific gene expression in lymphoid cells. Proc Natl Acad Sci USA 1990 ; $90: 11593-7$.

18. Murakami M, Narazaki M, Hibi $H$, Yamata H, Yasukawa K, Hamaguchi M, Taga T, Kishimoto T. Critical cytoplasmic region of the interleukin-6 signal transducer gp130 is conserved in the cytokine receptor family. Proc Natl Acad Sci USA $1991 ; 88$ : 11349-53.

19. Fukunaga $R$, Ishizada-Ikeda E, Pan CX Seto $Y$, Nagata $S$. Functional domains of the granulocyte colony-stimulating factor receptor. EMBO J1991; $10: 2855-65$.

20. Fukunaga R, Ishizaka-Ikeda E, Nagata S. Growth and differentiation signals mediated by different regions in the cytoplasmic domain of granulocyte colonystimulating factor receptor. Cell $1993 ; 74$ : 1079-87.

21. Ziegler SF, Bird TA, Morella KK, Mosley B, Gearing DP, Baumann $\mathrm{H}$. Distinct regions of the human granulocytecolony stimulating factor receptor cytoplasmic domain are required for proliferation and gene induction. Mol Cell Biol 1993; 13:2384-90.

22. Fukunaga $R$, Ishizada-Ikeda $E$ Nagata S. Purification and characterization of the receptor for murine granulocyte colony-stimulating factor. J Biol Chem 1990 $265: 14008-15$

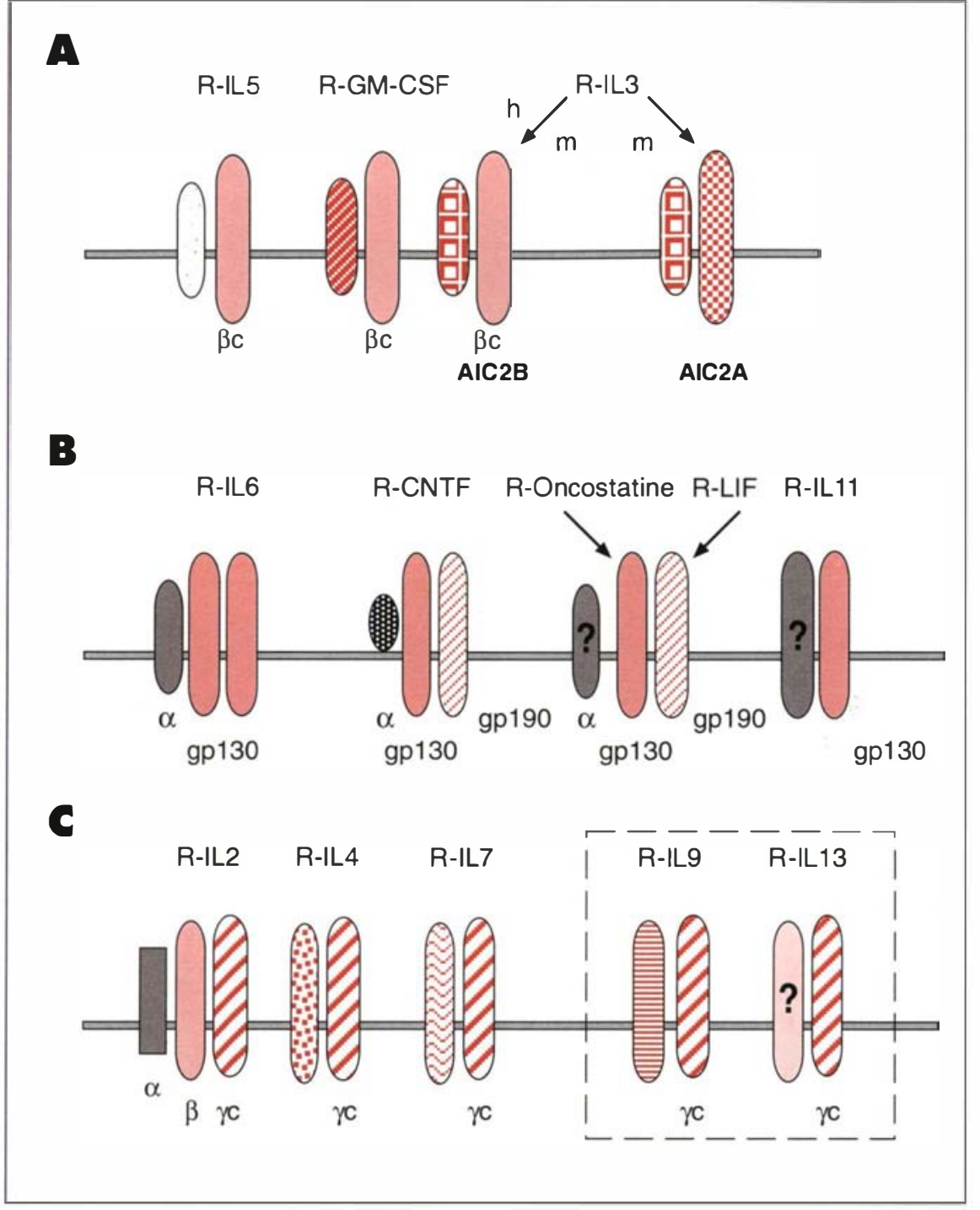

Figure 2. Chaînes communes aux récepteurs de cytokines. A. Les récepteurs de I'IL3, du GM-CSF et de I'IL5 comportent une chaîne $\alpha$ spécifique de chaque cytokine et une chaîne $\beta$ commune. Chez la souris, la chaîne $A / C 2 B$ peut s'associer à chacune des trois chaînes $\alpha$ alors qu'une deuxième chaîne $\beta$ AIC2A ne s'associe qu'à la chaîne $\alpha$ de l'IL3. II existe donc deux récepteurs de I'IL3 dans cette espèce. B. La gp130 est partagée entre les récepteurs de I'IL6, du CNTF, de l'oncostatine, du LIF et de I'IL 11. Cette chaîne ne fixe que l'oncostatine. L'IL6 et le CNTF se fixent sur une chaîne $\alpha$ spécifique. La chaîne fixant I'IL11 n'est pas clonée et l'on ignore si les récepteurs du LIF et de l'oncostatine comportent une chaîne $\alpha$ spécifique. C. La chaîne $\gamma$ du récepteur de I'IL2 est partagée par les récepteurs de I'IL2, I'IL4, I'IL7 et probablement I'IL9 et I'IL 13. La chaîne $\alpha$ du récepteur de I'IL2 (en rectangle) n'appartient pas, contrairement à toutes les autres chaînes, à la famille des récepteurs de cytokines. (D'après [53].) 
Il a été récemment rapporté que la gpl30 forme des homodimères après fixation de l'IL6 sur sa chaîne $\alpha$, alors que des hétérodimères ent re gpl30 et gpl90 sont formés après fixation du CNTF et du LIF sur leurs récepteurs respectifs [9, 10] (figure 2B). Si la distribution tissulaire des chaînes $\alpha$ de l'IL6 et du CNTF détermine la spécificité de réponse à ces cytokines, on ignore actuellement si les récepteurs de forte affinité du LIF et de l'oncostatine comportent également une chaîne $\alpha$.

Enfin, des récepteurs appartenant à un troisième groupe partagent une chaîne commune, la chaîne $\gamma \mathrm{du}$ récepteur de l'IL2. Le récepteur de l'IL2 est un complexe multimérique formé de trois chaînes : une chaîne $\alpha$, dont la séquence n'a pas de similitude avec celle des récepteurs de cytokines, et deux chaînes, $\beta$ et $\gamma$, qui appartiennent à cette famille de récepteurs et qui, toutes deux, sont indispensables à la transduction du signal biologique de l'IL2. Il avait été proposé que la chaîne $\gamma$ du récepteur de l'IL2 serait nécessaire à la fonction d'autres récepteurs de cytokines ayant pour cellules cibles des lymphocytes T. En effet, des mutations du gène de la chaîne $\gamma$, localisé chez l'homme en Xq13, sont responsables d'un déficit immunitaire héréditaire, le syndrome d'immunodéficience combinée sévère lié à l'X (XSCID pour X-linked severe combined immunodeficiency), alors que l'inactivation par recombinaison homologue du gène de l'IL2 chez la souris affecte peu le développement des lymphocytes $T$ [11]. Les mutations de la chaîne $\gamma$ du récepteur de l'IL2 observées dans les XSCID semblaient correspondre à un déficit global de réponse à un ensemble de cytokines nécessaires aux étapes précoces du développement lymphoïde $T$. On sait maintenant que cette chaîne est l'un des composants des récepteurs de l'IL7, de l'IL4, vraisemblablement de l'IL13 et peut-être de l'IL9 [12-14] (figure 2C). Cependant, bien que la chaîne $\gamma$ soit commune aux récepteurs de l'IL2, de l'IL4 et de l'IL7, ces cytokines activent des voies de signalisation différentes. La spécificité de signalisation observée en réponse à ces cytokines pourrait être conférée par la chaîne $\beta \mathrm{du}$ récepteur de
l'IL2, et les chaînes fixant l'IL4 et l'IL7.

Ces quelques exemples illustrent la complexité de structure et de fonctionnement des récepteurs de cytokines. Le bilan que l'on peut faire aujourd'hui de la structure de ces récepteurs n'est que provisoire et leur complexité est certainement plus grande qu'on ne l'avait initialement supposé. Par exemple, il est vraisemblable que plusieurs chaînes participent à la formation du récepteur de l'érythropoïétine (Epo). Une chaîne (p66) a été clonée qui fixe l'Epo avec une affinité comparable à celle observée pour les précurseurs érythroïdes normaux et qui participe à la signalisation par cette hormone [15]. Mais des expériences de pontage covalent ont montré l'association de l'Epo à deux chaînes supplémentaires de 85 et $100 \mathrm{kDa}$ dans les cellules érythroïdes et les cellules hématopoïétiques rendues sensibles à l'Epo après transfection de la p66 [16]. Si la p66 participe à la prolifération relayée par Epo [15], une étude récente utilisant des récepteurs chimères suggère que l'induction de gènes érythroïdes par Epo implique d'autres chaînes de ce récepteur [17]. On ne peut dès lors exclure que, comme les récepteurs de l'IL4 et de l'Epo, d'autres récepteurs de cette famille que l'on croit composés d'une seule chaîne ne soient, eux aussi, des complexes multimériques.

\section{Activation des récepteurs de cytokines : de multiples effets biologiques}

Les interleukines et les facteurs de croissance hématopoïétiques participent au contrôle de l'hématopoì̀se en agissant sur le couple prolifération-différenciation des cellules pluripotentes médullaires et des cellules progénitrices engagées vers une seule voie de différenciation, qui donnent ensuite naissance aux stades les plus différenciés des cellules sanguines. En plus de leurs cibles hématopoïétiques, certaines cytokines, notamment la famille IL6, ILl1, LIF, oncostatine dont les récepteurs sont exprimés sur de très nombreux tissus non hématopoïétiques, ont une action pléiotrope et sont responsables de signaux aussi différents que l'induction de différenciation neuronale cholinergique, la synthèse de protéines de la phase aiguë de l'inflammation par les cellules hépatiques ou l'altération du métabolisme lipidique dans le tissu adipeux [1]. Les cytokines dont les récepteurs appartiennent à la famille des récepteurs de cytokines sont généralement inhibiteurs de l'apoptose, inducteurs de la prolifération cellulaire et inducteurs ou inhibiteurs de la différenciation cellulaire. De toute évidence, la transduction de ces différents messages n'est pas indépendante. $\mathrm{Si}$ les voies métaboliques menant à la prolifération cellulaire commencent à être entrevues, celles contrôlant la différenciation des cellules et l'inhibition de l'apoptose restent encore indéterminées.

La transmission d'un signal biologique par les cytokines a été étudiée dans différents types cellulaires exprimant physiologiquement ces récepteurs, ou dans des cellules dépourvues de récepteurs fonctionnels et transfectées par une ou plusieurs chaînes polypeptidiques normales ou modifiées.

Un ensemble de données convergentes obtenues à partir des récepteurs tronqués a ainsi permis d'attribuer un rôle essentiel au domaine juxtamembranaire de la région cytoplasmique dans la prolifération cellulaire. Ce domaine, long de 60 à 100 acides aminés, inclut la région "riche en sérines " de la chaîne $\beta$ du récepteur de l'IL2, les régions "riches en prolines" des récepteurs de la GH et de la prolactine et comporte de courts motifs d'acides aminés relativement conservés, les boîtes 1 et 2 , initialement décrites pour la gp130 et le récepteur du G-CSF $[18,19]$. Le court domaine intracellulaire des chaînes $\alpha$ des récepteurs de l'IL3, du GM-CSF et de l'IL5, indispensable à la prolifération cellulaire, contient également un motif riche en prolines.

Les domaines des récepteurs de cytokines impliqués dans l'induction de gènes spécifiques et dans la différenciation cellulaire sont encore mal caractérisés. Pour le récepteur du G CSF, on sait cependant que, si la région juxtamembranaire du domai- 


\section{RÉFÉRENCES}

23. Taga $T$, Narazaki $M$, Yasukawa $K$, Saito T, Mikki D, Hamaguchi M, Davis S, Shoyab M, Yancopoulos GD, Kishimoto T. Functional inhibition of hematopoietic and neurotrophic cytokines by blocking the interleukin-6 signal transducer gp130. Proc Natl Acad Sci USA 1992 ; 89 : 10998-1001.

24. Dusanter-Fourt I, Casadevall $\mathrm{N}$, Lacombe C, Muller O, Billat C, Fischer S, Mayeux P. Erythropoietin induces the tyrosine phosphorylation of its own receptor in human erythropoietinresponsive cells. J Biol Chem 1992 ; 267 : 10670-5.

25. Harpur AG, Andres AC, Ziemiecki A, Aston RR, Wilks AF. JAK2, third member of the JAK family of protein tyrosine kinases. Oncogene $1992 ; 7$ : 1347-53.

26. Velasquez L, Fellous M, Stark GR, Pellegrini S. A protein tyrosine kinase in the interferon alpha/beta signaling pathway. Cell 1992 ; 70 : 313-22.

27. Witthuhn BA, Quelle FW, Silvennoinen $O$, Yi T, Tang B, Miura $O$, Ihle JH. JAK2 associates with the erythropoietin receptor and is tyrosine phosphorylated and activated following stimulation with erythropoietin. Cell 1993 ; $74: 227-36$.

28. Lütticken C, Wegenka UM, Yuan J, Buschmann J, Schindler C, Ziemiecki A, Harpur AG, Wilks AF, Yasukawa K, Taga T, Kishomoto T, Barbieri G, Pellegrini S, Sendtner M, Heinrich PC, Horn F. Association of transcription factor APRF and protein kinase Jakl with the interleukin-6 signal transducer gp 130 . Science $1994 ; 263$ : 89-92.

29. Stahl N, Boulton TG, Farruggella T, Ip NY, Davis S, Witthuhn BA, Quelle FW Silvennoinen O, Barbieri G, Pellegrini S Ihle JN, Yancopoulos GD. Association and activation of Jak-Tyk kinases by CNTF-LIFOSM-IL- 6 beta receptor components. Science $1994 ; 263: 92-5$.

30. Dusanter-Fourt I, Muller $O$ Ziemiecki A, Mayeux P, Drucker B, Djiane J, Wilks A, Harpur AG, Fischer S Gisselbrecht S. Identification of Jak protein tyrosine kinases as signaling molecules for prolactin. EMBO J1994; 11 : 2583-91.

31. Argetsinger LS, Campbell GS, Yang X, Witthuhn BA, Silvennoinen O, Ihle JN, Carter-Su C. Identification of JAK2 as a growth hormone receptor-associated tyrosine kinase. Cell 1993; $74: 23744$.

32. Kahn A. De la membrane au noyau, un couplage direct entre les récepteurs de cytokines et la machinerie transcriptionnelle. ne cytoplasmique est nécessaire à la prolifération, la région C-terminale règle de façon négative la prolifération et est indispensable à l'induction de gènes spécifiques (myéloperoxydase, élastase des neutrophiles) associés à la différenciation dans les cellules myéloïdes [20]. Une délétion du domaine C-terminal du récepteur du G-CSF pourrait être responsable de neutropénie congénitale sévère chez l'homme [21].

On a mis en évidence un certain nombre de relais intracellulaires, activés par la fixation des cytokines sur leurs récepteurs, et diverses études ont montré l'existence de voies de signalisation communes entre récepteurs de cytokines, récepteurs à activité tyrosine kinase et récepteurs des interférons.

La fixation des cytokines semble entraîner l'homo- ou l'hétérodimérisation (voire la multimérisation) de leurs récepteurs. Par analogie avec les récepteurs à activité tyrosine kinase intrinsèque, on peut envisager que l'oligomérisation constitue la première étape menant à l'activation des relais intracellulaires. Toutefois, le rôle du ligand n'est probablement pas limité à l'induction de la dimérisation. En effet, le récepteur du G CSF semble exister sous une forme multimérique en absence d'hormone [22]. Par ailleurs, des anticorps antigpl30 peuvent oligomériser cette protéine sans induire son activation [23].

Outre l'oligomérisation des récepteurs, le premier effet observé dans les cellules stimulées par des cytokines est l'augmentation de la phosphorylation de résidus tyrosine de différentes protéines cellulaires, et notamment du récepteur [8, 24]. Puisque ces récepteurs ne possèdent pas d'activité tyrosine kinase, il est nécessaire d'admettre que leur stimulation entraîne l'activation d'une ou de tyrosine kinase(s) associée(s). Dans l'état actuel de nos connaissances, plusieurs tyrosine kinases appartenant à deux familles différentes, les kinases de type Jak et les kinases de type Src, semblent activées par un même récepteur de cytokine. Cependant, les substrats exacts de ces kinases restent encore souvent mal connus.

\section{Un cocktail de tyrosine kinases activées}

Les kinases de la famille Jak comportent actuellement trois membres Jak1, Jak2 et Tyk2. Ces kinases présentent la particularité de contenir deux domaines kinases, l'un portant les motifs structuraux caractéristiques d'une activité tyrosine kinase, l'autre présentant des motifs d'une activité kinase encore indéfinie [25]. Par une approche génétique, Tyk2 a été identifiée comme nécessaire pour reconstituer une réponse cellulaire à l'interféron $\alpha$ dans une cellule mutante initialement résistante à cette cytokine [26]. Par analogie, et grâce également à la recherche systématique de tyrosine kinases susceptibles d'être impliquées dans le mécanisme d'action des récepteurs de cytokines, il a pu être montré que l'Epo, la GH, l'IL3, le GM-CSF, l'IL6, le LIF, l'OSM, le CNTF et la PRL induisent la phosphorylation de tyrosines et l'activation rapide et spécifique de tyrosine kinases de la famille Jak [27-30]. Le G-CSF activerait également des Jak et, pour l'IL2, de nouveaux membres de cette famille ont déjà été impliqués. L'activation de l'une ou l'autre de ces Jak ne semble pas spécifique d'une cytokine particulière (l'Epo, la GH, l'IL3, la PRL activent toutes Jak2) mais dépend parfois $d u$ contexte cellulaire. En effet, l'IL6 peut activer deux membres de la famille Jak qui peuvent être différents d'un type cellulaire à l'autre. Cette activation est indépendante de l'abondance des kinases Jak exprimées dans une cellule donnée et l'on ne connaît pas à l'heure actuelle les mécanismes contrôlant le choix de(s) membre(s) Jak(s) activé(s) [28]. Les tyrosine kinases de la famille Jak s'associent spécifiquement à la région cytoplasmique juxtamembranaire des récepteurs de l'Epo, du LIF (gpl90) et de la GH [27-29, 31] et en particulier à la boîte 1 du récepteur de l'Epo et de la GH. Exception faite du récepteur de la $\mathrm{GH}$, ces kinases sont préassociées au récepteur de cytokine, avant toute stimulation par le ligand, différentes Jak pouvant être préassociées à un même récepteur de cytokines. L'activation des Jak semble donc 


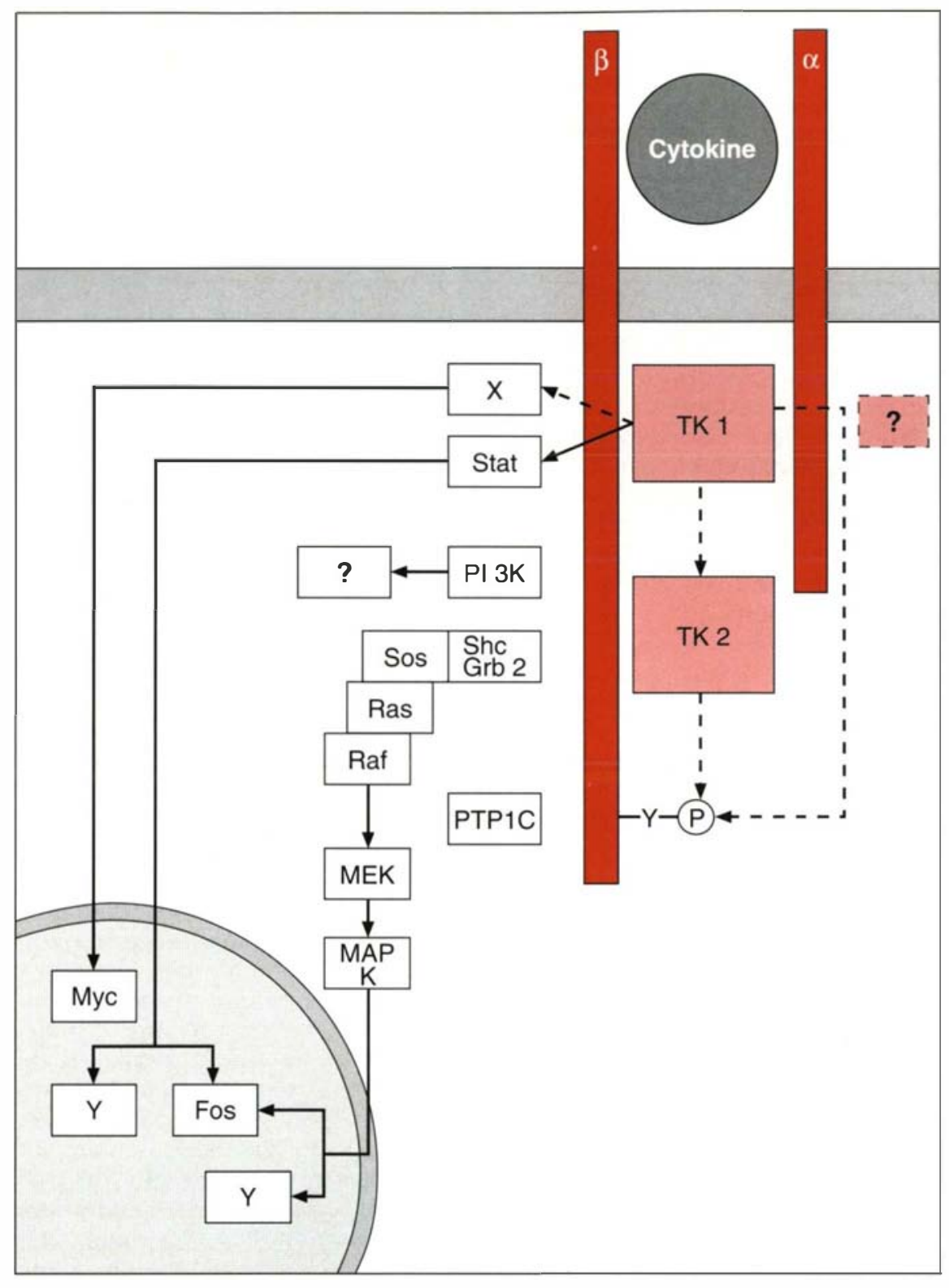

Figure 3. Mécanismes d'action intracellulaire des récepteurs de cytokines. (1) La fixation d'une cytokine sur la chaîne du récepteur assurant la spécificité de reconnaissance induit la formation d'un complexe multimérique par l'association d'une ou de plusieurs autres chaînes. (2) La formation de ce complexe entraîne l'activation de tyrosine kinases susceptibles de s'associer avec différentes régions de la chaîne $\beta$. Ces tyrosine kinases sont de plusieurs types. Les tyrosine kinases de la famille Jak reconnaissent la partie juxtamembranaire de ces récepteurs. Toutefois, nous ignorons encore si toute l'activité tyrosine kinase associée à cette région du récepteur et représentée sur ce schéma par "TK-1" est attribuable aux kinases de la famille Jak ou si d'autres kinases non encore identifiées interagissent aussi avec cette partie du récepteur. Les kinases de la famille Jak phosphorylent les facteurs de transcription de type Stat. Les cibles nucléaires de ces facteurs de transcription restent encore largement méconnues (Y). Parmi elles, se trouvent les gènes de certaines protéines de l'inflammation et le gène c-fos. La même région du récepteur est nécessaire à l'activation transcriptionnelle du gène c-myc. Cette activation met en jeu une activité tyrosine kinase dont nous ignorons encore si elle est différente de Jak et quels sont les relais impliqués dans cette activation $(X)$. Une région plus distale du récepteur est impliquée dans l'activation d'autres kinases (TK-2). Dans certains cas au moins, ces kinases appartiennent à la famille Src. La région de fixation de TK-1 (et peut-être l'activité TK-1 elle-même) est nécessaire à l'activation de TK-2. La même région du récepteur est impliquée dans l'activation de la phosphatidylinositol 3' kinase (PI3 K) et de la voie Ras/MAP kinases. De nombreux substrats des MAP kinases ont été identifiés, mais seule la voie menant à l'activation transcriptionnelle de c-fos est indiquée sur ce schéma. Le récepteur lui-même est généralement phosphorylé sur des résidus tyrosine, mais la kinase responsable de cette phosphorylation n'a pas encore été identifiée. La phosphorylation du récepteur pourrait créer des points de fixation pour un certain nombre de protéines de transduction dont la tyrosine phosphatase à domaines SH2, PTP1C, qui serait impliquée dans la remise au repos du récepteur. Enfin, des voies de signalisation mettant en jeu plus spécifiquement la chaîne $\alpha$ sont probables, bien qu'actuellement ces voies ne soient pas encore identifiées. Dans ce schéma, les voies de signalisation clairement établies sont en traits pleins alors que les relais supposés ou possibles sont en pointillés. 


\section{RÉFÉRENCES}

33. Hatekeyama M, Kono T, Kobayashi $N$ Kawahara A, Levin SD, Perlmutter RM Taniguchi $T$. Interaction of the IL-2 receptor with the $\mathbf{s r c}$-family kinase $\mathbf{p 5} 6^{\text {lck }}$ identification of novel intermolecular association. Science $1991 ; 252$ : 1523-8.

34. Hatakeyama M, Mori H, Doi $T$ Taniguchi T. A restricted cytoplasmic region of IL- 2 receptor beta chain is essential for growth signal transduction bu not for ligand binding and internalization. Cell $1989 ; 59: 837-45$.

35. Corey S, Eguinoa A, Puyana-Theall K Bolen JB, Cantley L, Mollinedo F Jackson TR, Hawkins PT, Stephen LR. Granulocyte macrophage-colony stimulating factor stimulates both association and activation of phosphoinositide $3 \mathrm{OH}$-kinase and src-related tyrosine kinase (s) in human myeloid derived cells. $E M B O$ J $1993 ; 12$ : 2681-90.

36. Moore B, Seroussi G, Jochim B Explorer de nouveaux domaines $\mathrm{SH} 2 / \mathrm{SH} 3$. médecine/sciences $1993 ; 9$ : 788-90.

37. Chardin P. Domaines SH2 et SH3 : un nouveau paradigme dans la transmission du signal, médecine/sciences $1994 ; 10: 709-12$.

38. Wang LM, Keegan AD, Paul WE, Heidaran MA, Gutkind S, Pierce JH. IL-4 activates a distinct signal transduction cascade from IL-3 in factor-dependent myeloid cells. EMBO ]1992; 11 : 4899-908.

39. Mayeux P, Dusanter-Fourt I, Muller O, Mauduit $P$, Sabbah $M$, Druker B, Vainchenker W, Fischer S, Lacombe C, Gisselbrecht S. Erythropoietin induces the association of phosphatidylinositol 3'-kinase with a tyrosine-phosphorylated protein complex containing the erythropoietin receptor. EurJ Biochem $1993 ; 216: 821-8$.

40. Gold MR, Duronio V, Saxena SP, Schrader JW, Aebersold R. Multiple cytokines activate phosphatidylinositol 3 kinase in hemopoietic cells. J Biol Chem 1994 ; 269 : 5403-12.

41. Yi T, Mui ALF, Krystal G, Ihle IN. Hematopoietic cell phosphatase associates with the interleukin-3 (IL-3) receptor $\beta$ chain and down-regulates IL-3-induced tyrosine phosphorylation and mitogenesis. Mol Cell Biol 1993 ; 13 : 7575-86.

42. De la Chapelle A, Traskelin AL, Juvonen E. Truncated erythropoietin receptor causes dominantly inherited benign human erythrocytosis. Proc Natl Acad Sci USA 1993 ; $90: 4495-9$.

43. Kahn A. La transmission du signal en amont et en aval de Ras. médecine/sciences $1992 ; 8: 1097-9$. être une conséquence de changements conformationnels affectant le complexe récepteur/Jak après la liaison du ligand. Il semble pourtant très probable que l'activation et la transphosphorylation des kinases Jak associées à chaque molécule de récepteur soient secondaires à la dimérisation/oligomérisation du récepteur après fixation du ligand. A l'appui de cette hypothèse, il a été démontré que la dimérisation de la chaîne $\beta$ du récepteur de l'IL6 (gp130) est nécessaire à la phosphorylation des Jak associées [28]. De même, la signalisation par les interférons $\alpha / \beta$ et $\gamma$ nécessite l'activation conjointe de deux Jak différentes, induisant une phosphorylation réciproque de résidus tyrosine (transphosphorylation) [32]. Il est probable qu'un mécanisme similaire soit mis en jeu pour les récepteurs de cytokines impliquant des Jak connues ou à découvrir

Puisqu'une même kinase de type Jak peut être activée par de multiples cytokines induisant des effets biologiques extrêmement variés, on peut penser que la spécificité de réponse cellulaire à une cytokine donnée sera déterminée par: (1) les types de substrats de cette kinase présents dans une cellule donnée; et/ou (2) les substrats associés à un récepteur donné ; et/ou (3) la combinatoire de Jak et d'autre(s) tyrosine kinase(s) spécifiquement associée(s) à chaque récepteur.

Différentes tyrosine kinases de la famille Src sont activées et semblent impliquées dans la transmission d'un signal biologique par les récepteurs de l'IL2 (Lck, Fyn, Lyn), de l'IL3 et du GM-CSF (Yes, Lyn), de l'IL7 (Fyn) et de l'oncostatine (Yes) [8, 33-35]. Dans le cas du récepteur de l'IL2, les domaines d'association entre la chaîne $\beta$ du récepteur et Lck ont été caractérisés et mettent en jeu la "région acide", située au centre du domaine intracellulaire de la chaîne $\beta$, d'une part, et le domaine kinase de Lck, d'autre part. L'activation de Lck nécessite cependant également la présence et l'intégrité de la région juxtamembranaire du domaine intracellulaire (riche en sérines) de la chaîne $\beta$ du récepteur de l'IL-2 [34]. Toutefois, cette interaction directe chaîne $\beta$-Lck ne serait pas indispensable à la transduction d'un signal prolifératif puisque la délétion de la région acide du récepteur $\beta$ n'empêche pas l'induction d'un signal mitogène par l'IL2 (voir plus loin).

\section{De multiples relais intracellulaires}

La liaison d'une cytokine à son récepteur déclenche la phosphorylation sur des tyrosines de tout un ensemble de protéines cellulaires qui sont actuellement en cours de caractérisation dans différents laboratoires. Les récepteurs eux-mêmes sont très généralement phosphorylés sur des tyrosines après activation. Toutefois, cette phosphorylation ne semble pas indispensable à la prolifération cellulaire puisque l'élimination des tyrosines de la partie intracellulaire des récepteurs de l'Epo, du G-CSF ou de la chaîne $\beta$ du récepteur de l'IL3 ne détruit pas leur potentialité de transduction d'un signal mitogène. A l'heure actuelle, aucun résidu tyrosine phosphorylé précis d'un récepteur de cytokines n'a encore été affecté à la liaison d'une molécule de signalisation donnée. Le rôle de cette phosphorylation générale des récepteurs de cytokines, induite par leur ligand respectif, reste encore indéterminé. Cependant, la phosphorylation de ces résidus tyrosine crée des sites d'ancrage potentiels de nombreuses molécules de signalisation contenant un domaine caractéristique de type SH2 (src homology 2) - comme cela a été décrit pour les récepteurs présentant une activité tyrosine kinase intrinsèque [ 36,37$]$ - qui pourraient intervenir dans l'induction de transcription de gènes spécifiques (maturation/différenciation cellulaire) ou simplement dans l'arrêt du signal d'activation cellulaire.

La phosphatidylinositol 3-kinase (PI3 kinase) est activée dans les cellules stimulées par l'Epo, l'IL2, l'IL3, l'IL4, l'IL5, l'IL7 ou le GM-CSF. Les mécanismes de cette activation restent obscurs ; ils pourraient mettre en jeu une association entre la sousunité régulatrice de cette enzyme (p85) et les tyrosines phosphorylées des récepteurs, des protéines de type IRS-1 (insulin receptor substrate 1), des kinases de la famille Src ou des protéines phosphorylées sur tyrosine 
dont l'identité reste à établir [35, 3840]. L'activation de cette enzyme pourrait jouer un rôle dans l'internalisation des récepteurs activés et/ou être impliquée dans la prolifération cellulaire.

Par ailleurs, la tyrosine phosphatase PTP1C est associée au récepteur de l'IL3 activé et vraisemblablement également au récepteur de l'Epo [41]. Cette enzyme pourrait être impliquée dans le processus de désactivation du récepteur. Un domaine de régulation a été décrit à l'extrémité C-terminale du récepteur de l'Epo dans une région riche en tyrosines. La délétion expérimentale de cette région se traduit in vitro par une hypersensibilité à l'Epo [15] et, chez l'homme, une mutation introduisant un codon stop prématuré éliminant les 70 acides aminés C-terminaux de l'EpoR est responsable d'érythrocytose familiale [42]. Cette région pourrait aussi fixer la phosphatase PTP1C, son élimination faisant alors disparaître un site d'inactivation du récepteur.

La voie de signalisation menant à l'activation des MAP kinases (mitogen activated protein kinases) et conduisant à l'expression des facteurs transcriptionnels c-Fos et c-Jun a été bien caractérisée pour les récepteurs à activité tyrosine kinase [43-45]. Cette voie est aussi utilisée par la plupart des récepteurs de cytokines. Elle fait intervenir le recrutement et/ou l'activation séquentielle de Shc/ Grb2, de facteurs d'échange de Ras tels Sos, de Ras, de Raf et des MAP kinases kinases (MEK) [46] (figure 3). Les kinases de la famille Src pourraient être impliquées dans l'activation de la voie Ras/MAP kinases [46]. En effet, la région " acide " de la chaîne $\beta$ du récepteur de l'IL2 nécessaire à l'activation de Lck est aussi nécessaire à l'activation de la voie Ras [47]. Dans le cas du récepteur du GM-CSF, une région située dans la partie médiane du domaine intracellulaire est aussi indispensable à l'activation de cette voie [48]. La délétion de ces régions n'entraîne pas la perte de réponse proliférative à l'IL2 ou au GM-CSF. Ainsi, la prolifération des cellules hématopoiétiques - au moins de certaines d'entre elles et contrairement aux fibroblastes - ne nécessiterait pas l'activation des MAP kinases.
Cependant, il a aussi été montré que des oligonucléotides antisens anti-craf inhibent la prolifération des cellules stimulées par l'IL3 [49]. Par ailleurs, il semble que les récepteurs tronqués incapables de stimuler cette voie de transduction ne puissent maintenir une prolifération cellulaire qu'en présence de sérum de veau fotal, c'est-à-dire dans des conditions permettant probablement une certaine activation de Ras et/ou de c-Fos [48]. L'importance de l'activation des MAP kinases dans la prolifération induite par les cytokines est donc encore mal comprise. Une toute nouvelle classe de molécules cytoplasmiques, dont la phosphorylation sur tyrosine est induite par de nombreuses cytokines, vient d'être identifiée. Il s'agit de facteurs transcriptionnels existant à l'état latent et inactifs dans le cytoplasme et dont la phosphorylation sur des tyrosines déclenche l'activation, c'est-à-dire leur association à d'autres chaînes polypeptidiques et/ou leur homodimérisation, leur translocation nucléaire ainsi que leur capacité à se lier à des séquences d'ADN spécifiques. Ces facteurs ont été d'abord identifiés par leur capacité à être activés par les interférons $(\alpha / \beta$ et $\gamma)$ et à induire la transcription de nombreux gènes contrôlés par les interférons [32]. Le facteur prototype, de $91 \mathrm{kDa}$ (p91), est activé par l'IFN $\gamma$ tandis que les trois sous-unités du facteur transcriptionnel ISGF3 $\alpha$ (p91, p84 et pl13) sont activées par l'IFN $\alpha / \beta$. Ces facteurs présentent une parenté de structure tout à fait intéressante. En particulier, ils contiennent de manière caractéristique un domaine d'homologie de type SH2 et de type SH3 dans leur partie C-terminale et une région de répétitions d'heptaleucine de type leucine zipper dans leur domaine $\mathrm{N}$-terminal pouvant être impliquée dans des interactions protéine-protéine (homo ou hétérotypiques). La phosphorylation sur des tyrosines de ces facteurs - et donc leur activation - est strictement dépendante de l'activation de kinases de la famille Jak. En revanche, cette activation est indépendante de l'activation de la voie de signalisation faisant intervenir Ras. Bien que la p9l contienne de nombreux résidus tyrosine, une seule tyrosine jouerait un rôle déterminant dans son activation. De plus, la présence d'un domaine $\mathrm{SH} 2$ fonctionnel est également nécessaire. Il a pu être montré que l'IL6, le LIF, l'OSM et le CNTF activent par phosphorylation sur des tyrosines la p9l et un nouveau membre de cette famille de facteurs transcriptionnels, APRF, immunologiquement proche mais différent de la p91 [28, 29]. De même, l'IL3/IL5/GM-CSF, l'IL4, l'Epo, la GH et la PRL activeraient de nouveaux membres de cette famille qu'il a été proposé d'appeler STAT pour signal transducers and activators of transcription [50-52]. Dans le cas des récepteurs comprenant la chaîne $\beta$ du récepteur de l'IL6 (gp130), la p9l est associée à la gp130 activée seulement si cette chaîne existe à l'état de dimère (gpl30-gpl 30 ou gpl30-gpl90). Ces facteurs pourraient donc être directement phosphorylés par les Jak au niveau d'un complexe récepteur/Jak/STAT. Cependant, la démonstration définitive de cet événement n'a pas encore été rapportée.

Ainsi, de manière générale, toute une panoplie de facteurs transcriptionnels de type STAT existerait à l'état latent dans le cytoplasme et serait activée par les kinases Jak. Chaque récepteur de cytokines pourrait activer une combinatoire spécifique de facteurs transcriptionnels STAT pouvant s'associer spécifiquement entre eux et/ou à d'autres facteurs de transcription conduisant à l'activation d'un ensemble de gènes communs et de gènes particuliers.

\section{Les cibles nucléaires des signaux induits par les cytokines sont encore bien mal connues}

Toutes les cytokines stimulant la prolifération cellulaire induisent l'expression transcriptionnelle du gène $c-m y c$. Le mécanisme de cette induction n'est pas encore précisé mais ferait intervenir la région juxtamembranaire des récepteurs de cytokines et impliquerait l'activation de tyrosine kinases [48]. Nous ignorons encore si ces tyrosine kinases sont les Jak kinases ou d'autres tyrosine kinases se fixant à la même région du récepteur. 


\section{RÉFÉRENCES}

44. Kaslauskas A. Receptor tyrosine kinases and their targets. Curr Op Gen Dev 1994; 4 : 5-14.

45. Boguski MS, McCornick F. Proteins regulating Ras and its relatives. Nature $1993 ; 366: 643-54$.

46. Chardin P. Protéines Ras et transmission des signaux mitogènes. médecine/sciences $1994 ; 10$ : $657-64$.

47. Satoh T, Minami Y, Kono T, Yamada K, Kawahara A, Taniguchi T, Kaziro Y. Interleukin-2-induced activation of ras requires two domains of interleukin-2 receptor $\beta$ subunit, the essential region for growth stimulation and Lck-binding domain. J Biol Chem 1992 ; 267 : 25423-7.

48. Sato N, Sakamaki K, Terada N, Arai K, Miyajima A. Signal transduction by the high-affinity GM-CSF receptor : two distinct cytoplasmic regions of the common $\beta$ subunit responsible for different signaling. EMBOJ 1993 ; 12 : 4181-9.

49. Carroll MP, Spivak JL, McMahon M, Weich N, Rapp UR, Stratford May W. Erythropoietin induces Raf-1 activation and Raf- 1 is required for erythropoietinmediated proliferation. I Biol Chem 1991 ; mediated pro

50. Larner AC, David M, Feldman GM, Igarashi K, Hackett RH, Webb DSA, Sweitzer SM, Petricoin III EF, Finbloom DS. Tyrosine phosphorylation of DNA binding proteins by multiple cytokines. Science 1993 ; $261: 1630-3$.

51. Kotanides H, Reich NC. Requirement of tyrosine phosphorylation for rapid activation of a DNA binding factor by IL-4. Science 1993 ; 262 : 1265-7.

52. Finbloom DS, Petricoin III EF, Hackett RH, David M, Feldman GM, Igarashi K, Fibach E, Weber MJ, Thorner MO, Silva CM, Larner AC. Growth hormone and erythropoietin differentially activate DNA-binding proteins by tyrosine phosphorylation. Mol Cell Biol 1994; 14 : 2113-8.

53. Kishimoto T, Taga T, Akira S. Cytokine
Par ailleurs, les facteurs transcriptionnels c-Fos et c-Jun sont induits par de nombreuses cytokines. Dans le cas de l'induction de $c$-fos, au moins deux types de facteurs transcriptionnels semblent impliqués. D'une part, des facteurs transcriptionnels de type STAT activés par l'IL6, l'Epo ou la GH se fixent sur les séquences d'ADN SIE présentes en amont du gène $c$-fos et pourraient ainsi moduler son expression. D'autre part, les facteurs de type Ets (Elk1) coopèrent avec SRF3 (serum response factor) pour stimuler la transcription via l'activation d'un complexe se fixant aux séquences SRE situées juste en aval des séquences SIE participent à l'induction de c-fos et sont activés par les MAP kinases comme cela a été rapporté pour de nombreux récepteurs de cette famille [53].

En revanche, en dehors de certains gènes bien caractérisés et spécifiquement induits par l'IL6, il existe très peu de gènes cellulaires pour lesquels des éléments de réponses spécifiques à certaines cytokines ont été définis. Dans le cas des gènes des protéines de phase aiguë induits par l'IL6, deux types de facteurs transcriptionnels sont également mis en jeu, des facteurs transcriptionnels de type STAT (p91/APRF) activés par phosphorylation sur tyrosine par le biais des kinases Jak et des facteurs de type NF-IL6 activés par le biais des MAP kinases [53].

Ainsi, de manière générale, deux voies d'activation, celle des facteurs transcriptionnels de type STAT, indépendante de l'activation d'un signal de type Ras, et celle des facteurs transcriptionnels activables par les MAP kinases et dépendant de l'activation de Ras, pourraient également être impliquées dans la transcription de gènes communs ou spécifiquement induits par une cytokine.

Bien que préliminaires, ces données suggèrent que les multiples effets biologiques d'une même cytokine, qui varient en fonction d'un contexte cellulaire et apparaissent même parfois contradictoires, sont relayés par une combinatoire de transmissions de signaux dont la nature varie en fonction de l'état de différenciacion des cellules cibles

\section{Summary}

Cytokin receptors and signal transduction

Cytokines (interleukins and colony stimulating factors) are involved in the proliferation and maturation of hematopoietic cells and in the regulation of immune responses. These effects are mediated through the high affinity binding of cytokines to specific cell surface receptors. Cloning of the cDNAs encoding cytokine receptors has revealed that most of them display common structural features in their extracellular domain and do not contain kinase consensus sequences in their cytoplasmic region. This rapidly growing cytokine receptor superfamily includes not only hematopoietin receptors but also receptors for growth hormone $(\mathrm{GH})$, prolactin and the ciliary neurotrophic factor (CNTF). Unlike growth factor receptors with intrinsic tyrosine kinase activity, cytokine receptors are often composed of several subunits. Cytokines mediating similar effects on the same target cells have been shown to share a common receptor chain essential for signal transduction. Thus, one receptor subunit is common to the IL3, GM-CSF and IL5 receptors, and the gp130, initially described as the IL6 receptor signal transducing chain, is one of the components of the IL11, LIF, oncostatin and CNTF receptors. Unexpectedly, however, the receptors for cytokines exhibiting different biological activities on T cells like IL2, IL 4 and IL7 also share a common chain and inherited mutations of the chain common to these three cytokine receptors are responsible for the human $\mathrm{X}$ linked severe combined immunodeficiency syndrome (XSCID). Although none of the cytoplasmic domains of cytokine receptors contains a tyrosine kinase motif, stimulation of target cells with cytokines induces the tyrosine phosphorylation of specific cellular proteins, suggesting that cytoplasmic tyrosine kinases interact with cytokine receptors. Several tyrosine kinases of the Src 


\begin{abstract}
Summary (suite)
family are known to be activated by cytokine receptors. Binding of Lck to the cytoplasmic domain of the IL2 receptor b chain occurs in a region of the receptor also responsible for Ras activation. However, this region is dispensable for IL2induced proliferation. More recently, it was shown that cytokines activate tyrosine kinases of the Jak family which interact with a region of the cytokine receptors proximal to the membrane and required for mitogenesis. This family of tyrosine kinases is involved in IFNa and g signal transduction, phosphorylating cytoplasmic transcription factors that translocate to the nucleus and induce the expression of IFN responsive genes. However, cytokines such as erythropoietin, IL3, GH and prolactin, exerting quite different biological activities, all activate the Jak2 kinase. Biological specificity may depend on the substrate specificity of the Jak kinase according to the cell type, on the activation of other unknown Jak kinases or of other tyrosine kinases, and/or on the activation of other signalling pathways such as Ras/MAP kinase, phosphatidyl inositol 3' kinase and their potential substrates.
\end{abstract}

\title{
TIRÉS À PART
}

S. Gisselbrecht.

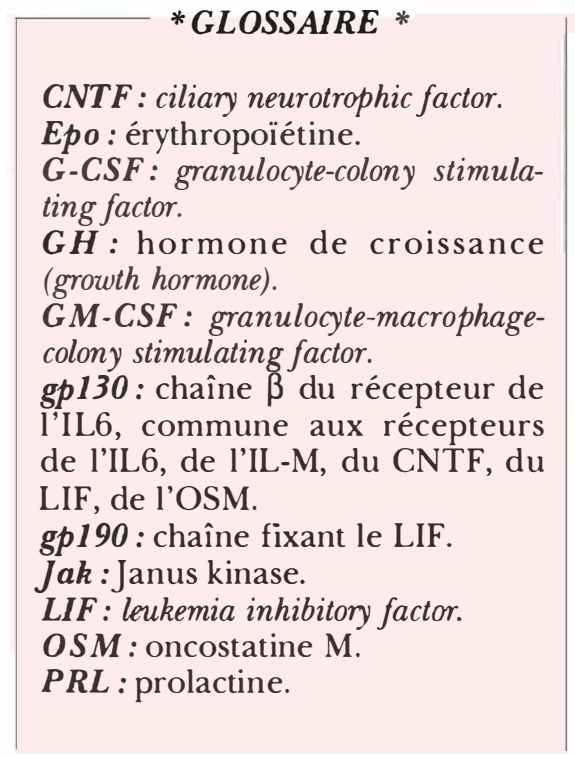

